\title{
RELATIVE LINEAR SETS AND SIMILARITY OF MATRICES WHOSE ELEMENTS BELONG TO A DIVISION ALGEBRA*
}

\author{
BY \\ M. H. INGRAHAM AND M. C. WOLF $\dagger$
}

It is the purpose of this paper to develop the theory of the similarity transformation for matrices whose elements belong to a division algebra. In order to get a basis for generalization, the theory of the similarity transformation for matrices whose elements belong to a field is sketched in what seems to the authors a more suggestive method than those used heretofore. $\ddagger$

L. A. Wolf's paper entitled Similarity of matrices in which the elements are real quaternions $\S$ treats the case of the quaternion algebra over any subfield of the real field, by passing to the equivalent square matrices of order $2 n$ with elements in the subfield.

Some of the results of the present paper are given in an abstract and a subsequent paper by $\mathrm{N}$. Jacobson.. $\mid$ Jacobson's results are to a certain extent more general. In the present paper a usable rational process is given for determining the equivalence or non-equivalence of matrices whose elements belong to a division algebra, and a theorem is developed concerning the rank of a polynomial in a matrix, which is not indicated by Jacobson. Some of the results contained herein were given by Ingraham at the summer meeting of the Society in 1935 . I

\section{THE SIMILARITY TRANSFORMATION FOR THE COMMUTATIVE CASE}

1. Review of certain known theory. If $M$ is an $m \times n$ matrix (i.e., a matrix with $m$ rows and $n$ columns) with elements in a field $F$, the rank of $M$ is

* Presented to the Society, September 13, 1935 and September 3, 1936; received by the editor s July 1, 1936 and December 21, 1936.

$\dagger$ The participation of Dr. Wolf in carrying out her research in connection with this paper was made possible by a grant from the Graduate School of the University of Wisconsin.

‡ For somewhat similar treatment see $\mathrm{F}$. Gantmacher, La théorie géométrique des diviseurs élémentaires d'après $M$. Krull, Transactions, University of Odessa, Mathematics 1, 1935, pp. 89-108 (Russian) avec Franz, resumé.

$\S$ L. A. Wolf, Bulletin of the American Mathematical Society, vol. 42 (1936), pp. 737-743.

$\|$ N. Jacobson, On pseudo-linear transformations, Proceedings of the National Academy of Sciences, vol. 21 (1935), pp. 667-670. N. Jacobson, Pseudo-linear transformations, Annals of Mathematics, vol. 38 (1937), p. 485.

T M. H. Ingraham, Characteristic spaces associated with the matrix whose elements belong to a division algebra, Bulletin of the American Mathematical Society, vol. 41 (1935), p. 622. 
equal to the maximum number of linearly independent rows and (which is the same) the maximum number of linearly independent columns. Also the rank of $M$ is $n-s$ where $s$ is the maximum number of linearly independent vectors $\xi$ satisfying the equation $M \xi=0$. The equivalence of the two definitions of rank is essentially the theorem that the order of the linear extension of the columns of $M$ plus the order of the maximal set right orthogonal to $M$ is equal to the number of columns of $M$.

The set $U$ of all vectors $\xi$ such that $M \xi=0$ is a linear set over $F$. $\xi$ and $U$ are both said to be right orthogonal to $M$. Let $L\left(\xi_{1}, \xi_{2}, \cdots, \xi_{n}\right)$ denote the least linear set containing $\xi_{1}, \xi_{2}, \cdots, \xi_{n}$, i.e., the totality of all vectors of the form $\sum \xi_{i} a_{i}$ where the $a_{i}$ are in the field $F . L\left(\xi_{1}, \xi_{2}, \cdots, \xi_{n}\right)$ is said to be the linear extension of $\xi_{1}, \xi_{2}, \cdots, \xi_{n}$.

2. Definition of relative linear independence, relative linear extensions, and connection with similarity transformation. Let $M$ be an $n \times n$ square matrix with elements in a field $F$. A set of vectors $\xi_{1}, \xi_{2}, \cdots, \xi_{k}$ is defined to be linearly independent relative to $M$ if no one of the $\xi_{i}$ is the zero vector and if whenever a set of polynomials $h_{1}, h_{2}, \cdots, h_{k}$ with coefficients in $F$ exists such that $\sum_{i=1}^{k} h_{i}(M) \xi_{i}=0$, it implies $h_{i}(M) \xi_{i}=0$, (i). A set $U$ of vectors is said to be linear relative to $M$ if (1) whenever $\xi_{1}$ is in $U, h(M) \xi$ is in $U$ for every polynomial $h$ in $F$; (2) if $\xi_{1}$ and $\xi_{2}$ are in $U$, then $\xi_{1}+\xi_{2}$ is in $U$. Denote by $L_{M}\left(\xi_{1}, \xi_{2}, \cdots, \xi_{k}\right)$ the linear extension of $\xi_{1}, \xi_{2}, \cdots, \xi_{k}$ relative to $M$, i.e., the least set $U$ containing $\xi_{1}, \xi_{2}, \cdots, \xi_{k}$ which is linear relative to $M$. It is the totality of vectors of the form $\sum_{i=1}^{k} h_{i}(M) \xi_{i}$, where the $h_{i}$ are polynomials in $F$.

It should be noted that if $M=I$, these definitions reduce to the usual ones for linear independence, linear sets, and linear extensions.

The polynomial $h$ and the vector $\xi$ are said to be associated relative to the matrix $M$ if $h(M) \xi=0$. As in the ordinary theory of the minimum equation of a matrix there is associated with every vector $\xi$ relative to $M$ a unique polynomial $h$ of lowest degree with leading coefficient unity. This polynomial divides all others associated with the vector $\xi$ and is said to be minimally associated with the vector $\xi$ relative to $M$. Clearly, the minimum polynomial such that $h(M)=0$ is the least common multiple of all those associated with the various vectors $\xi$ of the $n \times n$ vector space with elements in $F$.

Consider two similar matrices $M$ and $N$. Then there exists a non-singular matrix $T$ such that $T^{-1} M T=N$. Let $\xi_{1}, \xi_{2}, \cdots, \xi_{k}$ be linearly independent relative to $M$, and if $h_{i}$ is minimally associated with $\xi_{i}$ relative to $M$, then the vectors $\zeta_{i}=T^{-1} \xi_{i}$ are linearly independent relative to $N$, and $h_{i}$ is the minimum polynomial associated with $\zeta_{i}$ relative to $N$. 
Conversely, if $M$ and $N$ are two $n \times n$ matrices, if $\xi_{1}, \xi_{2}, \cdots, \xi_{k}$ is a set of vectors linearly independent relative to $M$, if $\zeta_{1}, \zeta_{2}, \cdots, \zeta_{k}$ is a set of vectors linearly independent relative to $N$ such that $L_{N}\left(\zeta_{1}, \zeta_{2}, \cdots, \zeta_{k}\right)$ $=L_{M}\left(\xi_{1}, \xi_{2}, \cdots, \xi_{k}\right)$ is the total vector space, and if $h_{1}, h_{2}, \cdots, h_{k}$ are minimally associated with $\xi_{1}, \xi_{2}, \cdots, \xi_{k}$ respectively relative to $M$ and are also minimally associated with $\zeta_{1}, \zeta_{2}, \cdots, \zeta_{k}$ respectively relative to $N$, then $M$ is similar to $N$. If $P=\left(\xi_{1}, M \xi_{1}, \cdots, M^{m_{1}-1} \xi_{1}, \xi_{2}, M \xi_{2}, \cdots, M^{m_{2}-1} \xi_{2}, \cdots, \xi_{k}\right.$, $\left.M \xi_{k}, \cdots, M^{m_{k}-1} \xi_{k}\right)$, and

$$
\begin{aligned}
& Q=\left(\zeta_{1}, N \zeta_{1}, \cdots, N^{m_{1}-1} \zeta_{1}, \zeta_{2}, N \zeta_{2}, \cdots, N^{m_{2}-1} \zeta_{2}, \cdots, \zeta_{k},\right. \\
& \left.\checkmark \zeta_{k}, \cdots, N^{m_{k}-1} \zeta_{k}\right)
\end{aligned}
$$

where $m_{i}$ is the degree of $h_{i}$, then $P$ and $Q$ are non-singular. If $T=P Q^{-1}$ then $T^{-1} M T=N$.

3. Theory of relative linear independence and extension. Invariants of the similarity transformation. If $U$ is a linear set relative to $M$, and if $\xi_{1}-\xi_{2}$ is in $U$, then it is said that $\xi_{1} \equiv \xi_{2} \bmod U$. The usual processes associated with congruences pertain to this definition. Moreover, given the vector $\xi$ and $U$, there exists a minimum polynomial $h$ with leading coefficient unity such that $h(M) \xi \equiv 0 \bmod U$ and this polynomial divides all other polynomials satisfying this congruence. The polynomial $h$ is said to be minimally associated with the vector $\xi \bmod U$ relative to $M$. If $h$ is associated with $\xi \bmod U$, then $h$ is associated with $\xi \bmod U_{1}$ for all relative linear sets $U_{1}$ containing $U$.

The following five theorems are given for two vectors but clearly, by iteration, lead to obvious generalizations for more than two vectors. In all of these the equalities may be replaced by congruences with a relative linear set as modulus.

THEOREM 1. If $h_{1}$ and $h_{2}$ are minimally associated with the vectors $\xi_{1}$ and $\xi_{2}$ respectively relative to $M$, and if $h_{1}$ and $h_{2}$ are relatively prime, then the vectors $\xi_{1}$ and $\xi_{2}$ are relatively linearly independent with respect to the matrix $M$.

Let $g_{1}(M) \xi_{1}=g_{2}(M) \xi_{2}=\xi_{3}$. The polynomial minimally associated with $\xi_{3}$ must be a divisor of both $h_{1}$ and $h_{2}$ and is therefore 1 . Hence if $g_{1}(M) \xi_{1}$ $=g_{2}(M) \xi_{2}$, then $g_{1}(M) \xi_{1}=g_{2}(M) \xi_{2}=0$, and the theorem is proved.

THEOREM 2. If $h_{1}$ and $h_{2}$ are two relatively prime polynomials whose product $h=h_{1} h_{2}$ is minimally associated with the vector $\xi$ relative to $M$, and if $\xi_{1}=h_{2}(M) \xi$ and $\xi_{2}=h_{1}(M) \xi$, then $\xi_{1}$ and $\xi_{2}$ are relatively linearly independent with respect to $M$ and $L_{M}\left(\xi_{1}, \xi_{2}\right)=L_{M}(\xi)$. Moreover, the polynomials $h_{1}$ and $h_{2}$ are minimally associated with the vectors $\xi_{1}$ and $\xi_{2}$ respectively relative to $M$. 
By Theorem 1 these vectors, $\xi_{1}$ and $\xi_{2}$, are relatively linearly independent with respect to $M$. Let $p_{1} h_{1}+p_{2} h_{2}=1$. Hence

$$
\xi=p_{2}(M) h_{2}(M) \xi+p_{1}(M) h_{1}(M) \xi=p_{2}(M) \xi_{1}+p_{1}(M) \xi_{2} .
$$

Hence $L_{M}\left(\xi_{1}, \xi_{2}\right)$ contains $L_{M}(\xi)$. Obviously $L_{M}(\xi)$ contains $L_{M}\left(\xi_{1}, \xi_{2}\right)$, and therefore $L_{M}(\xi)=L_{M}\left(\xi_{1}, \xi_{2}\right)$.

By a similar method one can prove

THEOREM 3. If $h_{1}$ and $h_{2}$ are relatively prime polynomials and are minimally associated with $\xi_{1}$ and $\xi_{2}$ respectively relative to $M$, then the polynomial minimally associated with $\xi_{1}+\xi_{2}$ relative to $M$ is $h_{1} h_{2}$; moreover $L_{M}\left(\xi_{1}+\xi_{2}\right)=L_{M}\left(\xi_{1}, \xi_{2}\right)$.

Suppose $h_{1}$ and $h_{2}$ are any two polynomials. One may express $h_{1}=\prod p_{i}^{s_{i}} \prod q_{i}^{t_{i}}$ and $h_{2}=\prod p_{i}^{v_{i}} \Pi q_{i}^{u_{i}}$ where the $p_{i}^{\prime}$ 's and $q_{i}$ 's are polynomials which are relatively prime, pair by pair, and where $s_{i} \geqq v_{i}$ while $t_{i}<u_{i}$. If $h_{1}$ and $h_{2}$ are minimally associated with the vectors $\xi_{1}$ and $\xi_{2}$, then by Theorem 2 vectors $\xi_{3}$ and $\xi_{4}$ exist with which are minimally associated the polynomials $g_{1}=\prod p_{i}^{s_{i}}$ and $g_{2}=\prod q_{i}^{u_{i}}$ respectively, and by Theorem 3 the polynomial minimally associated with $\xi_{3}+\xi_{4}$ is $g_{1} g_{2}$, the least common multiple of $h_{1}$ and $h_{2}$. One may therefore state

THEOREM 4. If $h_{1}$ and $h_{2}$ are minimally associated with the vectors $\xi_{1}$ and $\xi_{2}$ respectively relative to $M$, then there exists a vector $\xi$ contained in $L_{M}\left(\xi_{1}, \xi_{2}\right)$ such that the least common multiple of $h_{1}$ and $h_{2}$ is the polynomial minimally associated with $\xi$ relative to $M$.

THEOREM 5. If $h_{1}$ and $h_{2}$ are minimally associated with the vectors $\xi_{1}$ and $\xi_{2}$ respectively relative to $M$, if $h_{1}=p_{1} h_{2}$, and if $h_{3}$ is minimally associated with $\xi_{2} \bmod L_{M}\left(\xi_{1}\right)$ relative to $M$ so that $h_{3}(M) \xi_{2}=h_{4}(M) \xi_{1}$, then $p_{2}$ and $p_{3}$ exist such that $h_{2}=p_{2} h_{3}$ and $h_{4}=p_{3} h_{3}$; furthermore the vectors $\xi_{1}$ and $\xi_{3}=\xi_{2}-p_{3}(M) \xi_{1}$ are linearly independent relative to $M$, and $h_{3}$ is minimally associated with both $\xi_{3}$ and $\xi_{3} \bmod L_{M}\left(\xi_{1}\right)$ relative to $M$.

Since $h_{2}(M) \xi_{2}=0$, it follows that $h_{2}(M) \xi_{2} \equiv 0 \bmod L_{M}\left(\xi_{1}\right)$. Hence $p_{2}$ exists such that $h_{2}=p_{2} h_{3}$. Since $h_{3}(M) \xi_{2}=h_{4}(M) \xi_{1}, \quad 0=h_{2}(M) \xi_{2}=p_{2}(M) h_{3}(M) \xi_{2}$ $=p_{2}(M) h_{4}(M) \xi_{1}$, and hence $p_{2} h_{4}$ must be divisible by $h_{1}$. Moreover $h_{1}=p_{1} h_{2}=p_{1} p_{2} h_{3}$, hence $h_{4}$ is divisible by $p_{1} h_{3}$ and hence by $h_{3}$, and there exists a $p_{3}$ such that $h_{4}=p_{3} h_{3}$. Hence $h_{3}(M) \xi_{2}=p_{3}(M) h_{3}(M) \xi_{1}$, and so if $\xi_{3}=\xi_{2}-p_{3}(M) \xi_{1}$, then $h_{3}(M) \xi_{3}=0$. Suppose a polynomial $h_{5} \neq 0$ of lower degree than $h_{3}$ existed such that $h_{5}(M) \xi_{3}=0$, then $h_{5}(M) \xi_{2}=h_{5}(M) p_{3}(M) \xi_{1}$, that is, $h_{5}(M) \xi_{2} \equiv 0 \bmod L_{M}\left(\xi_{1}\right)$ where $h_{5}$ is of lower degree than $h_{3}$. Since this is impossible, $h_{3}$ is minimally associated with $\xi_{3}$ and with $\xi_{3} \bmod L_{M}\left(\xi_{1}\right)$. From 
this it can be proved at once that $\xi_{1}$ and $\xi_{3}$ are linearly independent relative to $M$.

From the discussion in the preceding part of this section it follows that given any set of vectors $\left(\zeta_{1}, \zeta_{2}, \cdots, \zeta_{k}\right)$, a set $\left(\xi_{1}, \xi_{2}, \cdots, \xi_{m}\right)$ may be found such that $\xi_{1}, \xi_{2}, \cdots, \xi_{m}$ are linearly independent relative to $M$ and such that $L_{M}\left(\xi_{1}, \xi_{2}, \cdots, \xi_{m}\right)=L_{M}\left(\zeta_{1}, \zeta_{2}, \cdots, \zeta_{k}\right)$. Moreover by repeated use of Theorems 2 and 5 and their extensions to more than two vectors and to congruences this may be done in such a way that the polynomials $h_{i}$ minimally associated with the $\xi_{i}$ may be taken as powers of irreducible polynomials, or by repeated use of Theorems 4 and 5 and their extensions, the $h_{i}$ may be chosen such that $h_{i+1}$ divides $h_{i}$. The only limitations on the $h_{i}$ are given by the following theorem.

THEOREM 6. If $\xi_{11}, \xi_{12}, \cdots, \xi_{18}$ and $\xi_{21}, \xi_{22}, \cdots, \xi_{2 t}$ are two sets of vectors each of which is linearly independent relative to $M$ such that $L_{M}\left(\xi_{11}, \xi_{12}, \cdots, \xi_{18}\right)$ $=L_{M}\left(\xi_{21}, \xi_{22}, \cdots, \xi_{2 t}\right)$, and if $h_{1 i}$ and $h_{2 i}$ are minimally associated with $\xi_{1 i}$ and $\xi_{2 i}$ respectively relative to $M$, and if $h$ is an irreducible polynomial, and $k$ any positive integer, then the number of the polynomials $h_{1 i}$ divisible by $h^{k}$ is equal to the number of the polynomials $h_{2 i}$ divisible by $h^{k}$.

Let $S_{1}=L_{M}\left(\xi_{11}, \xi_{12}, \cdots, \xi_{1 s}\right)$ and $S_{2}=L_{M}\left(\xi_{21}, \xi_{22}, \cdots, \xi_{2 t}\right)$. Since $S_{1}=S_{2}$, the numbers of linearly independent vectors in $S_{1}$ and $S_{2}$ respectively which are orthogonal to $h(M)$ are equal. The number of linearly independent vectors of $S_{1}$ orthogonal to $h(M)$ is equal to the sum of the numbers of such vectors in the sets $L_{M}\left(\xi_{1 i}\right)$. If $h_{1 i}=p_{1 i} h$, then the $m$ vectors $p_{1 i}(M) \xi_{1 i}$, $M p_{1 i}(M) \xi_{1 i}, \cdots, M^{m-1} p_{1 i}(M) \xi_{1 i}$, where $m$ is the degree of $h$, are linearly independent and form a base for the set of vectors orthogonal to $h(M)$ in $L_{M}\left(\xi_{1 i}\right)$. If $h_{1 i}$ is not divisible by $h$, then no vector of $L_{M}\left(\xi_{1 i}\right)$ other than the zero vector is orthogonal to $h(M)$. Hence the number of polynomials $h_{1 i}$ or $h_{2 i}$ divisible by $h$ is $n_{1} / m$, where $n_{1}$ is the order of the set of vectors in $S_{1}$ (equal to the order in $S_{2}$ ) orthogonal to $h(M)$. This proves the theorem for $k=1$.

Let $n_{2}$ be the number of linearly independent vectors $\xi$ in $L_{M}\left(h(M) \xi_{11}\right.$, $\left.\cdots, h(M) \xi_{18}\right)=L_{M}\left(h(M) \xi_{21}, \cdots, h(M) \xi_{2 t}\right)$ which satisfy the equality $h(M) \xi=0$, then by reasoning as above, $n_{2} / m$ is the number of polynomials $h_{1 i}$ or the number of polynomials $h_{2 i}$ which are divisible by $h^{2}$, etc.

As an immediate consequence follows

THeOREм 7. If $\xi_{1}, \xi_{2}, \cdots, \xi_{k}$ are linearly independent relative to $M$ such that $L_{M}\left(\xi_{1}, \xi_{2}, \cdots, \xi_{k}\right)$ is the total vector space, and if the polynomial $h_{i}$ minimally associated with $\xi_{i}$ relative to $M$ is a power of an irreducible polynomial, then these $h_{i}$ are the same for all sets of vectors satisfying these conditions. 
The polynomials of such a set are called the characteristic divisors of $M-\lambda I$, and if the irreducible polynomials are linear, the characteristic divisors of $M-\lambda I$ are identical with the elementary divisors as usually defined.

The invariance of the invariant factors also follows at once. The Dickson rational canonical form is a matrix $N$ such that the $h_{i}$ are the invariant factors when $\zeta_{1}, \zeta_{2}, \cdots, \zeta_{k}$ in $Q$ of equation (1) are $\delta_{1}, \delta_{m_{1}+1}$, $\delta_{m_{1}+m_{2}+1}, \cdots, \delta_{m_{1}+m_{2}+\cdots+m_{k-1}+1}$, where the vector $\delta_{j}$ is the Kronecker delta vector, 1 in the $j$ th place and zero elsewhere, and $m_{i}$ is the degree of $h_{i}$. It is also easy to characterize similarly the Jordan canonical form based on elementary divisors, as well as forms based on the highest power of irreducible factors occurring in the invariant factors, namely, the characteristic divisors.

The above treatment has two distinct advantages. First, starting with any $n$ linearly independent vectors, a set of vectors $\xi_{1}, \xi_{2}, \cdots, \xi_{k}$ linearly independent relative to $M$, such that $L_{M}\left(\xi_{1}, \xi_{2}, \cdots, \xi_{k}\right)$ is the total vector space, may be found by rational means, usually without great effort. Secondly, all of the canonical forms are derived by the same theoretical development.

\section{OUTLINE OF THE PROBLEM FOR THE CASE WHERE THE ELEMENTS} OF THE MATRIX BELONG TO A QUASI-FIELD $A$

The chief reason that the extension of the above theory to the case where the elements of $M$ belong to a quasi-field instead of a field is not trivial is that the rank of a polynomial in $M$ is not invariant under the similarity transformation. Moreover, one cannot say that if $g(M) \xi=0$ then $g\left(T^{-1} M T\right) T^{-1} \xi=0$.

In order to obviate these difficulties, it was found that a new operation instead of ordinary multiplication should be defined. Given the polynomial $g(\lambda)=\sum \lambda^{i} a_{i}$, the matrix $M$, and the vector $\xi$, we define $g(M) \odot \xi$ to be the vector $\sum M^{i} \xi a_{i}$. In the case that all the elements involved belong to a field, $g(M) \odot \xi=g(M) \xi$. From this definition it follows that if $g(M) \odot \xi=0$, $g\left(T^{-1} M T\right) \odot T^{-1} \xi=0$. Whenever $g(M) \odot \xi=0, \xi$ is said to be $\odot$-orthogonal (tentatively read as dot-orthogonal) to $g(M)$. It is seen that the order of the set of vectors $\odot$-orthogonal to $g(M)$ is an invariant of the similarity transformation. It does not follow if $g(M) \odot \xi=0$ that $g(M) \odot \xi a=0$, if $a$ is a non-commutative element of $A$. Secondly, the operation $\odot$ cannot be performed upon $\xi$ with only a knowledge of the matrix $g(M)$ but requires that one knows both $M$ and the polynomial $g$. Moreover, if $g_{1}=\sum \lambda^{i} a_{1 i}, g_{2}=\sum \lambda^{i} a_{2 i}$, $g_{1} \odot g_{2}$ is defined to be $\sum_{i} \lambda^{i} g_{2} a_{1 i}=\sum_{k} \lambda^{k} \sum_{i+j=k} a_{2 i} a_{1 j}$. This last definition is nothing but the usual one for the product $g_{2} g_{1}$ of two polynomials in a commutative indeterminate with coefficients in $A$. If $g=g_{1} \odot g_{2}$, then $g_{2}$ is said to be an interior factor of $g$, and $g_{1}$ an exterior factor of $g$. 
Dickson* has shown the existence of polynomials $p_{1}$ and $p_{2}$ such that $p_{1} \odot g_{1}+p_{2} \odot g_{2}$ is the greatest common interior divisor, $\left(g_{1}, g_{2}\right)$, of $g_{1}$ and $g_{2}$, and Ore $\dagger$ has proved the existence of the least common exterior multiple, $\left[g_{1}, g_{2}\right]$, of $g_{1}$ and $g_{2}$. The degree of $\left[g_{1}, g_{2}\right]$ is equal to the sum of the degrees of $g_{1}$ and $g_{2}$ less the degree of $\left(g_{1}, g_{2}\right)$.

In a quasi-field $A$ the total set of elements each of which is commutative with every other element of $A$ is a field containing 0 and 1 and is called the centrum $C$.

THEOREM 8. If $f_{1}$ and $f_{2}$ are two polynomials such that $f_{1} \odot f_{2}=h$, where $h$ is a polynomial with coefficients in the centrum, then $f_{1} \odot f_{2}=h=f_{2} \odot f_{1}$.

Let $h=f_{1} \odot f_{2}$, then $f_{1} \odot f_{2} \odot f_{1}=h_{1} \odot f_{1}=f_{1} \odot h$. Hence from the uniqueness of division, $h=f_{2} \odot f_{1}$.

In terms of the $\odot$-process relative linear independence and the extension process $L_{M}$ may readily be defined and it is not difficult to see that the theory of similarity transformation may be made to depend on the existence of sets of vectors $\xi_{1}, \xi_{2}, \cdots, \xi_{k}$ and $\eta_{1}, \eta_{2}, \cdots, \eta_{k}$ such that $L_{M}\left(\xi_{1}, \xi_{2}, \cdots, \xi_{k}\right)$ $=L_{N}\left(\eta_{1}, \eta_{2}, \cdots, \eta_{k}\right)$ is the whole vector space, and such that both sets of vectors are minimally associated with the same set of polynomials, as in the last section.

\section{VECTORS ORTHOGONAL TO POLYNOMIALS IN A MATRIX WITH ELEMENTS IN A FINITE DIVISION ALGEBRA $A$}

A quasi-field $A$ is a division algebra over the centrum $C$ if there exists a finite number $m$ of elements $\alpha_{i}$ in $A$ such that every element $a$ of $A$ can be expressed as

$$
a=\sum_{i=1}^{m} \alpha_{i} c_{i}
$$

where the coefficients $c_{i}$ are in $C$.

If $M$ is an $n \times n$ matrix and $\xi_{1}, \xi_{2}, \cdots$ a set of vectors with elements in a division algebra, the existence of a polynomial $g$ such that $g(M) \odot \xi_{i}=0$ can readily be established from the fact that not more than $n$ of the vectors $M^{i} \xi_{i}$ $(j=1,2, \cdots)$ are right linearly independent. If $g(M) \odot \xi_{i}=0$, then $g$ and $\xi_{i}$ are said to be associated relative to $M$. There is a unique polynomial $g_{i}$ of lowest degree with leading coefficient unity associated with $\xi_{i}$. This $g_{i}$ is an

* L. E. Dickson, Algebren und ihre Zahlentheorie, Zurich, 1927, p. 256. The product $g_{1} \odot g_{2}$ of this paper should be interpreted as $g_{2} g_{1}$ of Dickson and interior factor as left-hand factor, etc.

$\dagger$ O. Ore, Theory of non commutative polynomials, Annals of Mathematics, (2), vol. 34 (1933), pp. $480-508$. 
interior factor of every other polynomial $g$ associated with the vector $\xi_{i}$ and it is said to be minimally associated with $\xi_{i}$ relative to $M$.

A set of vectors is said to be right linear if for every $a_{1}$ and $a_{2}$ in $A \xi_{1} a_{1}+\xi_{2} a_{2}$ is in the set whenever $\xi_{1}$ and $\xi_{2}$ are in the set. In particular, if $a_{1}$ and $a_{2}$ are in the centrum $C$, the set of vectors is said to be linear relative to the centrum. It follows that

THEOREM 9. The maximal set of vectors $S$ associated with the polynomial $g$ relative to $M$ is a linear set relative to the centrum.

If $g=\sum \lambda^{i} a_{i}$ and $a$ is any element of $A$, let $g_{a}=\sum \lambda^{i} a^{-1} a_{i} a$, then $g_{a}$ will be called the transform of $g$ by $a$. $^{*}$ In most applications $a$ will be one of the basal elements $\alpha_{i}$ of $A$ relative to its centrum. Clearly the transform of a $\odot$-product of two polynomials is the $\odot$-product of their transforms. Moreover, if $\xi$ is associated with $g$, then $\xi a$ is associated with $g_{a}$, and if $g$ is minimally associated with $\xi$, then $g_{a}$ is minimally associated with $\xi a$.

THEOREM 10. If $h$ is a polynomial with coefficients in the centrum, the maximal space associated with h relative to $M$ is a right linear space; conversely, if $S$ is any right linear space, the polynomial minimally associated with the entire space $S$ relative to $M$ has coefficients in the centrum.

To prove the converse, let $a$ be any element of $A$ different from zero. $S a=S$. Let $g=\sum \lambda^{i} a_{i}$ be the polynomial of lowest degree associated with every vector of $S$, hence $g(M) \odot S=0$. Then $g_{a}(M) \odot S=g_{a}(M) \odot(S a)=0$. The degrees of $g_{a}$ and $g$ are equal, and the leading coefficient of each is unity. As the polynomial minimally associated with $S$ is unique, $g_{a}=g$ and $a^{-1} a_{i} a=a_{i}$, (i). Hence every $a_{i}$ is in the centrum $C$.

THEOREM 11. If $g$ is any polynomial with leading coefficient unity, there exists a matrix $M$ and a vector $\xi$ such that $g$ is minimally associated with $\xi$ relative to $M$.

If

$$
g=\lambda^{k}-\sum_{i=0}^{k-1} \lambda^{i} a_{i}
$$

consider the matrix

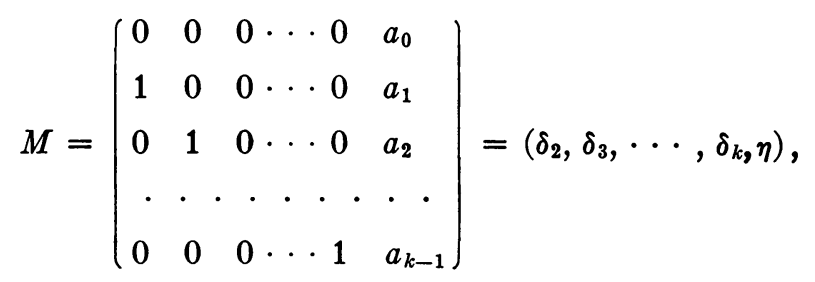

* This transform $g_{a}$ is a special case of the transforms of a polynomial $g$ by a second polynomial as used by Ore. In this paper, however, the only polynomials used for transforming others are constants. 
where $\delta_{i}$ is the Kronecker $\delta$ and $\eta$ is the $k$ th column of $M$. Then $\delta_{1}$ is effective as $\xi$.

TheOREm 12. If $g$ is any polynomial in the division algebra $A$, then the least common exterior multiple of $g$ and its transforms by the basal elements $\alpha_{i}$ of $A$, i.e., $\left[g, g_{\alpha_{2}}, \cdots, g_{\alpha_{m}}\right]$, is a polynomial with coefficients in the centrum.

From the preceding theorem there exists a matrix $M$ and a vector $\xi$ such that $g$ is minimally associated with $\xi$ relative to $M$. Any element in $U$, the right linear extension of $\xi$, is of the form $\xi\left(\sum \alpha_{i} c_{i}\right)$, where the $c_{i}$ are in the centrum. Since $g_{\alpha_{i}}$ is minimally associated with $\xi \alpha_{i}, h=\left[g, g_{\alpha_{2}}, \cdots, g_{\alpha_{m}}\right]$ is minimally associated with $U$, and hence by Theorem $10, h$ has coefficients in the centrum.

If $h=\left[g, g_{\alpha_{2}}, \cdots, g_{\alpha_{m}}\right]$, then $h$ is the polynomial of lowest degree with coefficients in the centrum which has $g$ as an interior factor, and $g$ is said to define $h$. If $h$ is the polynomial of lowest degree with coefficients in the centrum which is associated with $\xi$ relative to a matrix $M$, then $h$ is said to be minimally associated with $\xi$ over $C$ relative to $M$. It follows that if $h_{1}$ is a polynomial in $C$ and is associated with $\xi$ and if $h$ is minimally associated with $\xi$ over $C$, then $h$ is a factor of $h_{1}$. Also, if any polynomial $g$ is a factor of $h_{1}$, and if $g$ defines $h$, then $h$ is a factor of $h_{1}$.

THEOREM 13. If $g$ is minimally associated with $\xi$ relative to $M$, and if $g$ defines $h$, then $h$ is minimally associated with $\xi$ over the centrum relative to $M$.

THEOREM 14. If $g$ is an irreducible polynomial in $A$, then

$$
h=\left[g, g_{\alpha_{2}}, \cdots, g_{\alpha_{m}}\right]
$$

is a polynomial irreducible in the centrum $C$.

Suppose $h=h_{1} \odot h_{2}$, where $h_{1}$ and $h_{2}$ are in $C$, then $g$ divides either $h_{1}$ or $h_{2}$ for if this were not the case, polynomials $p_{1}, q_{1}, p_{2}, q_{2}$ exist such that $p_{1} \odot h_{1}=1+q_{1} \odot g$ and $p_{2} \odot h_{2}=1+q_{2} \odot g$, and hence

$$
p_{1} \odot h_{1} \odot p_{2} \odot h_{2}=p_{1} \odot p_{2} \odot h_{1} \odot h_{2}=1+\left(q_{1}+q_{2}+q_{1} \odot g \odot q_{2}\right) \odot g
$$

which would necessitate $g$ and $h_{1} \odot h_{2}$ being relatively prime, contrary to hypothesis.

THEOREM 15. If $g$ defines $h$ but no interior factor of $g$ of lower degree than the degree of $g$ defines $h$, and if $h$ is irreducible in $C$, then $g$ is irreducible in $A$.

THEOREM 16. If $g_{1}$ defines $h_{1}$, if $g_{2}$ defines $h_{2}$, and if $g_{1} \odot g_{2}$ defines $h$, then $h$ is a factor of $h_{1} \odot h_{2}$ and $\left[h_{1}, h_{2}\right]$ is a factor of $h$.

Let $h_{1}=f_{1} \odot g_{1}$ and $h_{2}=f_{2} \odot g_{2}$. By Theorem 8 


$$
h_{1} \odot h_{2}=f_{1} \odot g_{1} \odot f_{2} \odot g_{2}=f_{2} \odot f_{1} \odot g_{1} \odot g_{2},
$$

and hence $h$ is a factor of $h_{1} \odot h_{2}$. If $h=f \odot g_{1} \odot g_{2}$, then also from Theorem 8, $\left[h_{1}, h_{2}\right]$ is a factor of $h$.

The following two theorems are corollaries of the above theorem.

THEOREM 17. If $g=g_{m} \odot g_{m-1} \odot \cdots \odot g_{1}$ where the $g_{i}$ are irreducible, and if $h_{1}, h_{2}, \cdots, h_{t}$ are distinct polynomials in $C$ where $s_{j}$ of the $g_{i}$ each define $h_{j}$, $s_{j}>0$, and if $g$ defines $h$, then $h$ divides $\prod h_{j}^{\delta_{i}}$ and is divisible by $\prod h_{j}$.

THEOREM 18. If $g_{1}$ defines $h_{1}$ and $g_{2}$ defines $h_{2}$, and if $\left(h_{1}, h_{2}\right)=1$, then $g_{1} \odot g_{2}$ defines $h_{1} \odot h_{2}$.

THEOREM 19. If $g_{1}$ defines $h_{1}$, if $h_{2}$ divides $h_{1}$, and if $g_{2}=\left(g_{1}, h_{2}\right)$, then $g_{2} d e-$ fines $h_{2}$.

Suppose that $g_{2}$ defines $h_{3}$. By hypothesis $g_{2}$ is an interior factor of $h_{2}$, hence $h_{3}$ is a factor of $h_{2}$. Let $h_{2}=h_{4} \odot h_{3}, h_{1}=h_{5} \odot h_{2}=h_{5} \odot h_{4} \odot h_{3}$, and $g_{1}=g_{3} \odot g_{2}$. From Theorem 16, $g_{3}$ must define a multiple of $h_{5} \odot h_{4}$, call it $h_{6} \odot h_{5} \odot h_{4}$. Let $h_{6} \odot h_{5} \odot h_{4}=g_{4} \odot g_{3}$. If $h_{4}$ is of degree greater than zero, $g_{3}$ and $h_{4}$ must have a common interior divisor other than 1 , for if $\left(g_{3}, h_{4}\right)=1$ then there exist polynomials $p_{1}$ and $p_{2}$ such that $p_{1} \odot g_{3}+p_{2} \odot h_{4}=1$. Consequently, $g_{3}$ is an interior divisor of $h_{6} \odot h_{5}$ which is impossible. Let $g_{5}$ be the common factor of $g_{3}$ and $h_{4}$. From this it follows that $g_{5} \odot g_{2}$ is an interior factor of $g_{1}$ and of $h_{2}$, contrary to hypothesis.

If in Theorems 1-4 of parts I and II it is understood that all coefficients of the polynomials $h_{i}$ are in the centrum, these theorems apply to the case in which the basic number system is a division algebra rather than a field. These same modifications apply to the proofs of these theorems except that the polynomials $g_{1}$ and $g_{2}$ in the proof of Theorem 1 may not have coefficients in the centrum. Furthermore, the polynomial $h_{i}$ minimally associated with $\xi_{i}$ relative to a matrix $M$ is interpreted to be the polynomial with coefficients in the centrum minimally associated with $\xi_{i}$ relative to $M$ throughout these theorems.

TheOREm 20. If $g$, any polynomial in $A$, defines $h$, and if $h$ is associated with $\xi$ over the centrum relative to $M$, then there exists a set of vectors $\xi_{i}$, where $\xi_{i}$ is associated with $g_{\alpha_{i}}$, such that $\xi=\sum_{i=1}^{m} \xi_{i}$.

Let $h=f_{\alpha_{i}} \odot g_{\alpha_{i}}$. The polynomials $f, f_{\alpha_{2}}, \cdots, f_{\alpha_{m}}$ have no common exterior factor other than 1 , for if there were such, let it be $f_{1}$. Then $h=f_{1} \odot h_{1}$ where $h_{1}$ is a common multiple of $g$ and its transforms and is of lower degree than $h$. Hence there exist polynomials $p_{i}$ such that $\sum_{i} f_{\alpha_{i}} \odot p_{i}=1$. Let $\xi_{i}=f_{\alpha_{i}}(M) \odot p_{i}(M) \odot \xi$ and the theorem follows at once. Moreover, $\xi_{i} \alpha_{i}^{-1}$ is associated with $g$. 
THEOREM 21. If $U$ is a relative linear set and if $h$ is minimally associated with a vector $\xi \bmod U$ over the centrum $C$ relative to $M$, and if $g$ defines $h$, but no interior factor of $g$ of lower degree than the degree of $g$ defines $h$, then there exists a vector $\eta$ in $L_{M}(\xi)$ such that: (1) $g$ is minimally associated with $\eta \bmod U$, and (2) there exists a polynomial $p$ such that $g(M) \odot \eta=p(M) \odot h(M) \odot \xi$.

Consider $\xi_{1 i}=\xi_{i} \alpha_{i}^{-1}$ where the $\xi_{i}$ is defined as in the proof of Theorem 20. Let $h_{i}$ be minimally associated with $\xi_{1 i} \bmod U$ over $C$. Since $h$, which is minimally associated with $\xi \bmod U$, is associated with each $\xi_{i} \bmod U, h$ both divides and is divisible by $\left[h_{1}, h_{2}, \cdots, h_{m}\right]$, hence $\left[h_{1}, h_{2}, \cdots, h_{m}\right]=h$. For any polynomial $q$ and vector $\xi$,

$$
(q(M) \odot \xi) \alpha_{i}^{-1}=q_{\alpha_{i}^{-1}}(M) \odot\left(\xi \alpha_{i}^{-1}\right) .
$$

Also there exists a polynomial $q_{1}$ such that

$$
q_{\alpha_{i}^{-1}}(M) \odot\left(\xi \alpha_{i}^{-1}\right)=q_{1}(M) \odot \xi .
$$

Hence for some $q_{2}$,

Hence

$$
\begin{aligned}
\xi_{1 i} & =\left(f_{\alpha_{i}}(M) \odot p_{i}(M) \odot \xi\right) \alpha_{i}^{-1} \\
& =f(M) \odot p_{i \alpha_{i}{ }^{-1}}(M) \odot\left(\xi \alpha_{i}^{-1}\right) \\
& =f(M) \odot q_{2}(M) \odot \xi .
\end{aligned}
$$

$$
g(M) \odot \xi_{1 i}=h(M) \odot q_{2}(M) \odot \xi,
$$

so that any relative linear combination $\eta$ of the $\xi_{1 i}$ whose coefficients are polynomials in $C$ will satisfy condition (2) of the theorem. The existence of such a combination $\eta$ with which $h$ is minimally associated is guaranteed by Theorem 4. This $\eta$ will also satisfy condition (1).

THEOREM 22. If $g_{1}$ and $g_{2}$ each defines $h$ but no interior divisor of $g_{1}$ or of $g_{2}$ of degree less than the degree of $g_{1}$ or $g_{2}$ respectively defines $h$, then $g_{1}$ and $g_{2}$ are of the same degree.*

Let $\langle g\rangle$ represent the degree of any polynomial $g$. Suppose $\left\langle g_{1}\right\rangle \leqq\left\langle g_{2}\right\rangle$. By Theorem 11 a matrix $M$ and a vector $\xi_{1}$ can be chosen such that the order of $M$ is equal to $\left\langle g_{1}\right\rangle$ and $g_{1}$ is minimally associated with $\xi_{1}$ relative to $M$. By Theorem 21 the existence of a vector $\xi_{2}$ is established such that $g_{2}$ is minimally associated with $\xi_{2}$ relative to $M$, but the polynomial minimally associated with any vector relative to $M$ is of degree not more than the order of $M$. Hence $\left\langle g_{1}\right\rangle=\left\langle g_{2}\right\rangle$.

Polynomials such as $g_{1}$ and $g_{2}$ of Theorem 22 are said to be polynomials of minimum degree defining $h$. The degree of $g_{1}$ and $g_{2}$ is dependent upon $h$

* This proof, independent of any theorem of unique factorization, seems of interest. 
alone, and the degree of such polynomials shall be denoted by the symbol $\langle\langle h\rangle\rangle$, that is, $\langle\langle h\rangle\rangle=\left\langle g_{1}\right\rangle=\left\langle g_{2}\right\rangle$.

THEOREM 23. If $h$ is irreducible in $C$ and if $g$ is a polynomial of minimum degree defining $h$, then the degree of $h$ is an integral multiple of the degree of $g$.

THEOREM 24. If $g_{1}$ defines $h_{1}$ and $g_{2}$ defines $h_{2}$, then there exists some transform $g_{2 \alpha_{i}}$ of $g_{2}$ such that $g_{2 \alpha_{i}} \odot g_{1}$ defines $h_{3}$ where $h_{1}$ is a factor of $h_{3}$ but $h_{3} \neq h_{1}$ and $h_{3}$ is a factor of $h_{2} \odot h_{1}$.

Suppose for every $i, g_{2 \alpha_{i}} \odot g_{1}$ defines $h_{1}$ and therefore is an interior factor of $h_{1}$. Then $\left[g_{2} \odot g_{1}, g_{2 \alpha_{2}} \odot g_{1}, \cdots, g_{2 \alpha_{m}} \odot g_{1}\right]=h_{2} \odot g_{1}$ is an interior factor of $h_{1}$, and therefore there exists a polynomial $f$ such that $h_{1}=f \odot h_{2} \odot g_{1}=h_{2} \odot f \odot g_{1}$. Hence $f \odot g_{1}$ is in the centrum, which contradicts the hypothesis.

One can readily obtain

THEOREM 25. If $h$ is an irreducible polynomial and if $g_{1}$ is a polynomial of minimum degree defining $h$, there exists a set of transforms $g_{i}(i=1,2, \cdots, t)$ of $g_{1}$ by the basal elements $\alpha_{i}$ such that $g_{t} \odot g_{t-1} \odot \cdots \odot g_{1}$ is a polynomial of minimum degree defining $h^{t}$, and hence $\left\langle\left\langle h^{t}\right\rangle\right\rangle=t\langle\langle h\rangle\rangle$.

TheOREM 26. If $\xi_{1}, \xi_{2}, \cdots, \xi_{k}$ is a set of vectors linearly independent relative to $M$ such that $L_{M}\left(\xi_{1}, \xi_{2}, \cdots, \xi_{k}\right)$ is the whole space, and if $g_{i}$ is the minimum polynomial associated with $\xi_{i}$ relative to $M$, then the rank of $h(M)$, where $h$ is any polynomial in the centrum, is equal to $m-\sum_{i=1}^{k}\left\langle g_{1 i}\right\rangle$ where $m$ is the order of $M$ and $g_{1 i}=\left(h, g_{i}\right)_{\mathrm{ex}}$, the greatest common exterior divisor of $h$ and $g$.

Since $g_{1 i}=\left(h, g_{i}\right)_{\mathrm{ex}}$, let $g_{i}=g_{1 i} \odot g_{2 i}$ and $h=g_{1 i} \odot g_{3 i}=g_{3 i} \odot g_{1 i}$. Hence $h(M) \odot g_{2 i}(M) \odot \xi_{i}=0$ and also $h(M) \odot M^{j} \odot g_{2 i}(M) \odot \xi_{i}=0$. Since $g_{i}$ is the minimum polynomial associated with $\xi_{i}$, the number of vectors $M^{i} \odot g_{2 i}(M) \odot \xi_{i}$ right linearly independent and orthogonal to $h(M)$ is equal to the degree of $g_{1 i}$, $\left\langle g_{1 i}\right\rangle$. Furthermore, since $\xi_{1}, \xi_{2}, \cdots, \xi_{k}$ are linearly independent relative to $M$ by hypothesis, all vectors of the form $M^{i} \odot g_{2 i}(M) \odot \xi_{i}$ for $i=1,2, \cdots, k$ and $j=0,1,2, \cdots,\left\langle g_{1 i}\right\rangle-1$ are right linearly independent and orthogonal to $h(M)$. Consequently it follows that the rank of $h(M)$ is $m-\sum_{i=1}^{k}\left\langle g_{1 i}\right\rangle$; for if any other vector $\xi$ were orthogonal to $h(M)$ it would be dependent upon those orthogonal to $h(M)$ in each of $L_{M}\left(\xi_{1}\right), L_{M}\left(\xi_{2}\right), \cdots, L_{M}\left(\xi_{k}\right)$.

IV. EXISTENCE FOR $n$-SPACE OF A BASE, LINEARLY INDEPENDENT RELATIVE TO AN $n \times n$ MATRIX

If $M$ is an $n \times n$ matrix, and if the minimum polynomial in $C$ of $M$ is $\prod h_{i}{ }^{k_{i}}$, where the $h_{i}$ are distinct irreducible polynomials, if a linearly independent base relative to $M$ were found for the space associated with $h_{i}{ }^{k_{i}}$, then a line- 
arly independent base relative to $M$ for the whole space is the totality of vectors in the bases for the separate spaces associated with each of the $h_{i}{ }_{i}$, according to Theorem 2 .

The rank of $\prod_{i \neq j} h_{i}{ }^{k_{i}}(M)$ is equal to the order of the space orthogonal to $h_{j}{ }^{k_{j}}(M)$. Hence the columns of $\prod_{i \neq j} h_{i}{ }^{k_{i}}(M)$ form a base for the space associated with $h_{j}{ }^{k_{j}}$. At least one of these columns is a vector such that $h_{j}{ }^{k_{j}}$ is minimally associated with it over $C$ relative to $M$ or else $h_{j}$ appears to a lower power than the $k_{j}$ th in the minimum polynomial of $M$ in $C$.

To simplify the notation, let $h^{k_{1}}$ be the highest power of an irreducible polynomial $h$ in $C$ occurring in the minimum polynomial of $M$ in $C$, and let $g_{1}$ be a polynomial of minimum degree defining $h$. By Theorem 24 there exist transforms, $g_{k_{1}}, g_{k_{1}-1}, \cdots, g_{1}$, of $g_{1}$ by certain basal elements $\alpha_{i}$ of $A$ such that $g_{k_{1}} \odot g_{k_{1-1}} \odot \cdots \odot g_{1}$ is a polynomial of minimum degree defining $h^{k_{1}}$. By the preceding paragraph and Theorem 21 there exists a vector $\xi_{1}$ such that $g_{k_{1}} \odot g_{k_{1-1}} \odot \cdots \odot g_{1}$ is the minimum polynomial associated with $\xi_{1}$, and $h^{k_{1}}$ is minimally associated over $C$ with $\xi_{1}$. Since $h^{t}(M) \odot \xi_{1}$ and $g_{t}(M) \odot g_{t-1}(M) \odot \cdots \odot g_{1}(M) \odot \xi_{1}$ are minimally associated with $g_{k_{1}-t} \odot g_{k_{1}-t-1} \odot \cdots \odot g_{1}$ and $g_{k_{1}} \odot g_{k_{1}-1} \odot \cdots \odot g_{t+1}$ respectively, the order of $L_{M}\left(h^{t}(M) \odot \xi_{1}\right)$ is equal to the order of $L_{M}\left(g_{t}(M) \odot g_{t-1}(M) \odot \cdots \odot g_{1}(M)\right.$ $\left.\odot \xi_{1}\right)$. These two spaces are identical since the first is contained in the second. Therefore, if $f_{1}$ is any polynomial such that $h^{k_{1}-t}(M) \odot f_{1}(M) \odot \xi_{1}=0$ there exist polynomials $p_{1}$ and $p$ such that

$$
\begin{aligned}
f_{1}(M) \odot \xi_{1} & =p_{1}(M) \odot h^{t}(M) \odot \xi_{1} \\
& =g_{t}(M) \odot g_{t-1}(M) \odot \cdots \odot g_{1}(M) \odot p(M) \odot \xi_{1} ;
\end{aligned}
$$

i.e., any vector in $L_{M}\left(\xi_{1}\right)$ orthogonal to $h^{k_{1}-t}(M)$ is of the form given above.

Suppose $k_{2} \leqq k_{1}$ is the largest integer such that there exists a vector $\xi_{21}$ in the space associated with $h^{k_{1}}$ for which $h^{k_{2}}$ is the minimum polynomial in $C$ associated with $\xi_{21} \bmod L_{M}\left(\xi_{1}\right)$. This vector $\xi_{21}$ may be found among the columns of $\prod_{i \neq j} h_{i}{ }^{k}(M)$ where $h=h_{j}$. Since $f=g_{k_{2}} \odot g_{k_{2}-1} \odot \cdots \odot g_{1}$ is a polynomial of minimum degree defining $h^{k_{2}}$, by Theorem 21 the existence of a vector $\xi_{22}$ is established such that $f$ is minimally associated with $\xi_{22} \bmod L_{M}\left(\xi_{1}\right)$, and also the existence of $p_{2}$ is assured such that $f(M) \odot \xi_{22}=h^{k_{2}}(M) \odot p_{2}(M)$ $\odot \xi_{21}$ and hence the vector $f(M) \odot \xi_{22}$ is in $L_{M}\left(\xi_{1}\right)$ and orthogonal to $h^{k_{1}-k_{2}}(M)$. If equation (2) is applied with $t=k_{2}$, there exists a polynomial $p_{3}$ such that $f(M) \odot \xi_{22}=f(M) \odot p_{3}(M) \odot \xi_{1}$. Take $\xi_{2}=\xi_{22}-p_{3}(M) \odot \xi_{1}$. It follows that $f(M) \odot \xi_{2}=0$, and since $\xi_{2} \equiv \xi_{22} \bmod L_{M}\left(\xi_{1}\right), f$ is minimally associated both with $\xi_{2}$ and with $\xi_{2} \bmod L_{M}\left(\xi_{1}\right)$.

Let $k_{3}$ be the largest integer such that there exists a vector $\xi_{31}$ in the space associated with $h^{k_{1}}$ such that $h^{k_{3}}$ is minimally associated over $C$ with 
$\xi_{31} \bmod L_{M}\left(\xi_{1}, \xi_{2}\right)$. The polynomial $h^{k_{1}}$ is associated with $\xi_{31}$, moreover $h^{k_{2}}$ is associated with $\xi_{31} \bmod L_{M}\left(\xi_{1}\right)$ because of the maximal property of $k_{2}$. Then in $L_{M}\left(\xi_{1}, \xi_{2}, \xi_{3}\right)$ there will be a $\xi_{3}$ minimally associated with $g_{k_{3}} \odot g_{k_{3}-1} \odot \cdots \odot g_{1}$ $\bmod L_{M}\left(\xi_{1}, \xi_{2}\right)$ and hence minimally associated with $h^{k_{3}} \bmod L_{M}\left(\xi_{1}, \xi_{2}\right)$ over $C$, and such that $h^{k_{3}}(M) \xi_{3}=0$.

This process may be continued to complete the base for the total vector space.

It has been proved by Theorem 26 that

$$
r k\left(h^{k}(M)\right)=m-\sum_{i}\left\langle\left(h^{k}, g_{k_{i}} \odot g_{k_{i}-1} \odot \cdots \odot g_{1}\right)\right\rangle .
$$

Hence

$$
\left.r k\left(h^{k}(M)\right)=m-k\langle\langle h\rangle\rangle \text { (number of } k_{i} \geqq k\right)-\sum_{i} k_{i}\langle\langle h\rangle\rangle
$$

where the sum runs for values $k_{i}<k$. From this it follows that the polynomials associated with the above canonical proper base of the relative set equal to the total vector space are completely determined by a knowledge of the rank of the powers of $h_{i}$, where the $h_{i}$ are the irreducible factors of the minimum equation of $M$. Since in part I it was shown that the matrices $M$ and $N$ are similar if relative to $M$ and $N$ there exists a proper base for the total vector space associated with the same set of minimum polynomials, Theorem 27 follows.

THEOREM 27. If $h=\prod_{i=1}^{l} h_{i}{ }^{t_{i}}$ is the minimum polynomial in the centrum for each of two matrices $M$ and $N$, where $h_{i}$ is irreducible, a necessary and suffcient condition that the matrix $M$ is similar to the matrix $N$ is that the rank of $h_{i}{ }^{j}(M)$ is equal to the rank of $h_{i}{ }^{j}(N)$ for every $i=1,2, \cdots, l$ and $j=1,2, \cdots, t_{i}$.

If $\xi_{i j},(i=1,2, \cdots),(j=1,2, \cdots)$, is a set of linearly independent vectors whose linear extension relative to $M$ is the whole space, if $g_{i j}$ is the minimum polynomial associated with $\xi_{i j}$, and if $g_{i j}$ is a polynomial of minimum degree defining $h_{i}{ }^{k_{i j}}$, such an $h_{i}{ }^{k_{i j}}$, where $h_{i}$ is irreducible, is called a characteristic divisor of $M$. Hence the necessary and sufficient condition that two matrices be similar is that the characteristic divisors of the two matrices be equal. It is useful to note that the above implies that two matrices $M$ and $N$ are similar if for every polynomial $h$ with coefficients in the centrum the rank of $h(M)$ is equal to the rank of $h(N)$.

Since two matrices are equivalent only if $r k\left(h^{k}(M)\right)=r k\left(h^{k}(N)\right)$ whenever $h$ is irreducible and $k$ is a positive integer, a necessary condition that for a system of polynomials $g_{i}$ there exists a set of vectors $\xi_{i}$ linearly independent relative to $M$ and such that the minimum polynomial associated with $\xi_{i}$ is $g_{i}$ and 
$L_{M}\left(\xi_{1}, \xi_{2}, \cdots, \xi_{k}\right)$ is the whole space, is that the numbers $\sum\left\langle\left(h^{k}, g_{i}\right)\right\rangle$ are those specified by Theorem 27. This is sufficient because a diagonal block matrix $N$ may be constructed with blocks of the form of the matrix in Theorem 11, having $g_{i}$ as the minimum polynomial of each block.

The remainder of this section is devoted to describing a process which furnishes a method of testing whether two matrices are similar, and if $M$ and $N$ are similar, it furnishes a method of finding a set of polynomials $g_{i}$ and sets of vectors $\xi_{i}$ and $\eta_{i}$, each $k$ in number, such that the $\xi_{i}$ are linearly independent relative to $M$ and the $\eta_{i}$ are linearly independent relative to $N$; such that $L_{M}\left(\xi_{1}, \xi_{2}, \cdots, \xi_{k}\right)=L_{N}\left(\eta_{1}, \eta_{2}, \cdots, \eta_{k}\right)=S$, the total space; and such that $g_{i}$ is minimally associated with $\xi_{i}$ relative to $M$, and $g_{i}$ is minimally associated with $\eta_{i}$ relative to $N$. This process is wholly rational and does not involve the factorization of polynomials in the centrum into irreducible factors nor knowledge of a polynomial of minimum degree defining $h$; any factorization into powers of relatively prime factors in the centrum $C$ of the minimum polynomial in $C$ of $M$ and $N$ is suitable for an initial start and for each of these factors it is sufficient to have some polynomial $g$ defining it. Either the required sets of vectors are found or one of the known factors of the minimum polynomial in $C$ of $M$ and $N$ is factored with coefficients in $C$, or else a polynomial $g$ of lower degree than the degree of the initial $g$ is found which is associated with some factor of this minimum polynomial. The method therefore either yields the required vectors and polynomials or it reduces in a finite number of steps to the case studied in the beginning of this section. It should be borne in mind that this process is to be carried out simultaneously for $M$ and $N$ in practice, but the following treatment is for a matrix $M$ only.

Let the minimum polynomial with coefficients in $C$ for both $M$ and $N$ be $\prod h_{i}{ }^{l_{i}}$, where the $h_{i}$ are relatively prime. The whole vector space is the sum of the relative linear spaces orthogonal to $h_{i}{ }^{l_{i}}$ and as above there is at least one vector such that $h_{i}{ }^{l_{i}}$ is minimally associated with it. These spaces are linearly independent relative to $M$. To simplify notation, consider $h$ to be any of the $h_{i}$, and $g_{i}$ a transform of $g_{1}$. If, without assuming that the polynomials involved are irreducible, the method used in the beginning of this section is applied in constructing a proper base relative to a matrix $M$ and a proper base relative to a matrix $N$, these bases having the properties described in the preceding paragraph, then the following situations may arise, and these are the only difficulties in applying this reasoning and process.

1. A vector $\xi$ and a linear set $U$ may be found such that $h^{k}(M) \odot \xi \neq 0$ $\bmod U$ and $h^{k+1}(M) \odot \xi=0 \bmod U$, but the polynomial minimally associated with $\xi \bmod U$ over $C$ is not $h^{k+1}$. This leads to a factorization of $h$. 
2. $g_{1}$ and a set of transforms $g_{2}, g_{3}, \cdots, g_{t}$ exist such that $g_{t} \odot g_{t-1} \odot \cdots \odot g_{1}$ defines $h^{t}$ but there exists a transform $g_{t+1}$ such that $g_{t+1} \odot g_{t} \odot \cdots \odot g_{1}$ defines a multiple of $h^{t}$, not equal to $h^{t}$, and not equal to $h^{t+1}$. This also leads to a factorization of $h$.

3. A vector $\xi$ and a linear set $U$ exist such that $g_{t} \odot g_{t-1} \odot \cdots \odot g_{1}$ defines $h^{t}$, and $h^{t}$ is minimally associated with $\xi$ over $C$ but $\xi$ is minimally associated with a proper divisor, $g_{11}$, of $g_{t} \odot g_{t-1} \odot \cdots \odot g_{1}$. In this case the polynomial $g_{11}$ defines $h^{t}$ where $\left\langle g_{11}\right\rangle\left\langle t\left\langle g_{1}\right\rangle\right.$. By Theorem $19\left(h, g_{11}\right)$ defines $h$. If $\left\langle\left(h, g_{11}\right)\right\rangle\left\langle\left\langle g_{1}\right\rangle\right.$ then one arrives immediately at a new start with a polynomial of lower degree than $\left\langle g_{1}\right\rangle$ defining $h$. If $\left\langle\left(h, g_{11}\right)\right\rangle \geqq\left\langle g_{1}\right\rangle$, let $g_{11}=g_{12} \odot\left(h, g_{11}\right)$. By Theorem 16, $g_{12}$ defines a power of $h$, less than or equal to the $(t-1)$ st power, whereas $\left\langle g_{12}\right\rangle\left\langle(t-1)\left\langle g_{1}\right\rangle\right.$. Continuing with $\left(h, g_{12}\right)$ defining $h$ and by repeated use of the preceding argument, in a finite number of steps a polynomial is found, of degree lower than $\left\langle g_{1}\right\rangle$ defining $h$. This case may arise in trying to find a vector $\xi$ minimally associated with $g_{t} \odot g_{t-1} \odot \cdots \odot g_{1}$, and also if for such a $\xi, h^{t_{1}}(M) \odot \xi$ is minimally associated with a proper divisor of $g_{t-t_{1}} \odot g_{t-t_{1}-1} \odot \cdots \odot g_{1}$.

In carrying out this process simultaneously for $M$ and $N$, one is led either to the proof of the non-similarity of $M$ and $N$ or the construction of proper bases for the whole space relative to $M$ and $N$ respectively, such that the polynomials minimally associated with the one base are equal to those minimally associated with the other.

This procedure which for the general case seems to be both long and involved is for such usual cases as third- and fourth-order matrices with quaternionic elements not very difficult to carry through. For practical operation it should be noted that if $\prod h_{i}{ }^{l_{i}}$ is the minimum polynomial of $M$, and the $h_{i}$ are relatively prime, then the columns of $\prod_{i \neq j} h_{i}{ }^{l_{i}}$ form a base for the space orthogonal to $h_{j}^{l_{j}}(M)$.

It would seem that other problems could be advantageously attacked by methods of this paper. As an example, given two similar matrices $M$ and $N$, all non-singular matrices $S$ such that $S^{-1} M S=N$ consist of matrices $S$ that transform $\left(\xi_{i}, M \xi_{i}, M^{2} \xi_{i}, \cdots, M^{\left\langle g_{i}\right\rangle-1} \xi_{i}\right)$ into $\left(\eta_{i}, N \eta_{i}, N^{2} \eta_{i}, \cdots, N^{\left\langle\xi_{i}\right\rangle-1} \eta_{i}\right)$, (i), where the $\xi_{i}$ and $\eta_{i}$ are sets of vectors whose relative linear extensions are the whole space and where $g_{i}$ is minimally associated with $\xi_{i}$ relative to $M$ and with $\eta_{i}$ relative to $N$. In particular, the non-singular matrices commutative with $M$ are those which transform any set $\xi_{i}$ into a set $\xi_{1 i}$, having the same property.

UNIVERSITY OF WISCONSIN, Madison, Wis. 